\title{
Despacho óptimo de potencia reactiva considerando un abordaje multiperíodo
}

\author{
Daniel C. Londoño Tamayo, Walter M. Villa-Acevedo, y Jesús M. López-Lezama \\ Facultad de Ingeniería, Dpto. de Ingeniería Eléctrica, Universidad de Antioquia, Grupo de investigación GIMEL, \\ Medellín-Colombia (correo-e: dcamilo.Iondono@udea.edu.co; walter.villa@udea.edu.co; jmaria.lopez@udea.edu.co)
}

Recibido May. 6, 2021; Aceptado Jul. 5, 2021; Versión final Ago. 16, 2021, Publicado Dic. 2021

\section{Resumen}

Este estudio tiene como objetivo proponer un nuevo modelo y método de solución para el despacho óptimo de potencia reactiva multiperíodo. Este es un problema intrínsecamente no convexo y no lineal; por lo tanto, se soluciona mediante la metaheurística conocida como optimización de mapeo de media varianza. El modelo propuesto permite incorporar restricciones intertemporales para limitar el número de operaciones. Una de las ventajas del modelo es el diseño de su función objetivo que permite validar de forma simultánea la factibiliad y optimalidad de una solución. Se utiliza el sistema de prueba IEEE de 30 barras para validar el modelo y método de solución. Los resultados permiten concluir que el modelo propuesto garantiza una adecuada gestión de la potencia reactiva que minimiza las pérdidas y que mantiene los movimientos de taps de transformadores y bancos de capacitores dentro de límites para garantizar su vida útil.

\section{Optimal reactive power dispatch considering a multi-period approach}

\begin{abstract}
The main objective of this study is to propose a new model and solution method for multi-period optimal reactive power dispatch. This is an inherently non-convex and nonlinear problem. Therefore, it is solved by applying a mean-variance mapping optimization metaheuristic. The proposed model allows incorporating intertemporal constraints. One advantage of the model is the design of its objective function that allows to simultaneously assess the feasibility and optimality of a solution. The model and solution approach is validated by using the IEEE 30-bus test system. The results show and allow concluding that the proposed model guarantees an adequate reactive power management that minimizes power losses by keeping transformer taps and capacitor bank movements within limits to maximize lifetime.
\end{abstract}




\section{INTRODUCCIÓN}

El despacho óptimo de potencia reactiva (DOPR) consiste en la gestión óptima de los recursos de generación de reactivos y control de tensión de un sistema de potencia. Este problema se lleva a cabo después de la programación del despacho de la potencia activa, y debe realizarse sin alterar la programación previa de esta en los generadores; con excepción del nodo de referencia que debe compensar las pérdidas (Gutiérrez et al., 2017). Aunque el objetivo principal del DOPR es la reducción de pérdidas, también se han considerado otros objetivos como el mejoramiento del perfil de tensiones, la minimización del desvío de tensiones con respecto a un valor dado y el mejoramiento de la estabilidad de tensión (Mohseni-Bonab y Rabie., 2017).

EI DOPR se puede plantear de forma monobjetivo o multiobjetivo y solucionar mediante metodologías de carácter clásico o metaheurístico. Las metodologías clásicas incluyen: programación lineal, programación cuadrática y métodos de descomposición (Davoodi, et al., 2020). Por otro lado, dentro de las técnicas metaheurísticas se pueden citar: algoritmos evolutivos (Yan et al., 2004), algoritmos de inteligencia de enjambre (Singh et al., 2015), algoritmos caóticos (Mugemanyi et al., 2020) y métodos híbridos (Jamal et al., 2020). Una clasificación de las técnicas de solución empleadas en el DOPR puede ser consultada en (Gutiérrez et al., 2016). El DOPR también se puede abordar de forma monoperiodo o multiperiodo. Este último conocido como despacho óptimo de potencia reactiva multiperiodo (DOPRM), el cual consiste en realizar el despacho de los recursos de potencia reactiva de manera multihoraria, teniendo en cuenta la variación en el tiempo de las fuentes de generación y las cargas conectadas al sistema. El DOPRM es una variante del DOPR, que busca gestionar los recursos de potencia reactiva de manera multihoraria, considerando las mismas variables de control del abordaje monoperiodo. Así mismo, el DOPRM puede tener en cuenta restricciones en el número de operaciones en un intervalo de tiempo dado (horarias y diarias).

En el DOPRM, la función objetivo más común corresponde a la reducción de pérdidas. Zhang et al., (2016) solucionan el DOPRM para minimización de pérdidas de potencia activa teniendo en cuenta pronósticos de generación distribuida y reduciendo las operaciones en bancos de capacitores. Zhao et al., (2015) proponen un modelo DOPRM con restricciones de estabilidad de tensión. En este caso la cantidad de reservas dinámicas de potencia reactiva se utiliza como medida de la estabilidad de tensión del sistema. La solución del DOPRM se lleva a cabo mediante un método híbrido que combina la técnica de ramificación y poda con un método de punto interior primal dual. Huang et al., (2019) proponen una coordinación entre las fuentes de generación distribuida conectadas a un sistema, y la inyección de potencia reactiva discretizada, con el objetivo de reducir pérdidas de energía. Este enfoque es comparado con otras metodologías, y brinda un esquema para reducción de pérdidas de potencia en un horizonte de tiempo de 24 horas. López et al., (2015) abordan el tema de planeación de despacho óptimo de reactivos. En este caso, los autores emplean una subfunción que compara las pérdidas de potencia activa en un tiempo dado, con el valor óptimo propuesto por el operador de red. Los ajustes de tomas de los transformadores se modelan como variables enteras, mientras las demandas activa y reactiva se consideran funciones de distribución de probabilidad. Theologi et al., (2017) presentan un análisis de pérdidas de energía mediante una subfunción que involucra los pasos y cambios de taps de bancos de capacitores y reactores, y otras dos subfunciones que involucran la potencia activa y reactiva inyectada en los nodos del sistema, respectivamente.

En este artículo se presenta una extensión multiperiodo del modelo de flujo óptimo reactivo propuesto por VillaAcevedo et al., (2018). Como novedad se incorporan restricciones intertemporales que limitan los movimientos de taps de transformadores y bancos de capacitores y se emplea la versión de enjambre del algoritmo MVMO (mean variance mapping optimization) como método de solución. Las pruebas desarrolladas en el sistema IEEE de 30 barras evidencian la aplicabilidad del modelo propuesto y la robustez del método de solución. Una de las principales ventajas del modelo propuesto, que lo diferencia de los modelos de DOPRM propuestos en la literatura técnica, es su función objetivo que consiste en una multiplicatoria de subfunciones diseñada de tal forma que su valor máximo es uno cuando se obtiene una solución factible desde el punto de vista operativo que además cumple con una meta de reducción de pérdidas establecida previamente por el operador de red.

\section{MODELO MATEMÁTICO DEL DOPRM}

El modelo matemático del DOPRM propuesto en este artículo es una extensión del propuesto por VillaAcevedo et al., (2018). La función objetivo que se ilustra en (1) es una multiplicatoria de diferentes subfunciones. Uno de los términos es una meta de reducción de pérdidas diaria indicada como $f_{\text {lossd. }}$ Los otros términos permiten un manejo alternativo de restricciones relacionadas con límites de tensión en los nodos del sistema, como también flujos y pérdidas de potencia por las líneas de transmisión. En el modelo propuesto se multiplican diferentes subfunciones exponenciales que evalúan una meta o restricción operativa y de maniobra en el sistema bajo análisis. La idea es que cada subfunción entregue un valor igual a 1 si se cumplen las condiciones o metas operativas propuestas. De este modo, el producto total de las subfunciones, en condiciones operativas factibles y cumpliendo con el valor meta de reducción de pérdidas debe ser igual a 1. 
En caso contrario, la función objetivo es menor que 1.

$$
\mathrm{FO}=\prod_{t=1}^{24}\left\{\prod_{i=1}^{N_{L}} f_{V N}(i, t) * \prod_{j=1}^{N_{K}} f_{C L}(j, t) * \prod_{k=1}^{N_{T}} f_{C T h}(k, t)\right\} * f_{\text {lossd }} *\left\{\prod_{k=1}^{N_{T}} f_{C T d}(k) * \prod_{l=1}^{N_{C}} f_{C C d}(l)\right\}
$$

En este caso, $f_{V N}(i, t)$ es la subfunción de tensión para la barra ien el periodo de tiempo $t, f_{C L}(j, t)$ es la subfunción de flujo de potencia de la línea $j$ en el periodo $t, f_{C T h}(k, t)$ es la subfunción de maniobras realizadas en el periodo t para el tap del $k$-ésimo transformador, $f_{C T h}(k)$ es la subfuncion de maniobras realizadas en un día por el transformador $\mathrm{k}$; finalmente, $f_{C C d}(l)$ es la subfunción de maniobras realizadas en un día por el l-ésimo banco de capacitores.

El horizonte de planeación considerado por defecto es de 24 horas; sin embargo, cualquier otro horizonte operativo puede ser considerado. En este caso, se debe tener la información correspondiente para cada periodo de tiempo. Por otro lado, la función objetivo descrita anteriormente obedece a un problema de optimización determinista. Se parte de la hipótesis de que se tiene una adecuada predicción de las variables de generación y demanda; por lo tanto, la estocasticidad asociada a las fuentes de generación o a la demanda no se ha tenido en cuenta.

\section{Restricciones de tensión, flujos y meta de pérdidas de potencia}

Las expresiones matemáticas de las subfunciones que controlan los límites de tensión, flujo de potencia en líneas y la meta de pérdidas están dadas por las ecuaciones (2), (3) y (4), respectivamente.

$$
\begin{aligned}
& f_{V N}(i, t)=\left\{\begin{array}{c}
e^{\lambda_{v}\left(V_{\max _{i}}-V_{i, t}\right)}, V_{i, t}>V_{\max _{i}} \\
e^{\lambda_{v}\left(V_{i, t}-V_{\min _{i}}\right)}, V_{i, t}<V_{\min _{i}} \\
1, V_{\min _{i}} \leq V_{i, t} \leq V_{\max _{i}}
\end{array}\right\} \\
& f_{C L}(j, t)=\left\{\begin{array}{c}
e^{\lambda_{b}\left(\operatorname{Load}_{R j_{\max }}-\operatorname{Load}_{R j, t}\right)}, \operatorname{Load}_{R j, t}>\operatorname{Load}_{R j_{\max }} \\
1, \quad \operatorname{Load}_{R j, t} \leq \operatorname{Load}_{R j_{\max }}
\end{array}\right\} \\
& f_{\text {lossd }}=\left\{\begin{array}{cc}
e^{\lambda_{l}\left(\text { Loss }_{R e f}-P_{\text {lossd })}\right.}, P_{\text {lossd }}>\operatorname{Loss}_{\text {Ref }} \\
1, & P_{\text {lossd }} \leq \operatorname{Loss}_{\text {Ref }}
\end{array}\right\}
\end{aligned}
$$

Donde $\lambda_{v}$ es una constante de penalización exponencial por desviación de tensión encontrada en cada nodo, $V_{\text {maxi }}$ es el voltaje máximo permitido en cada nodo $i$ en por unidad (p.u), $V_{\text {mini }}$ es el voltaje mínimo permitido en cada nodo $i$ en p.u, $V_{i, t}$ es el voltaje en cada nodo $i$ en el periodo $t ; \lambda_{b}$ es una constante de penalización

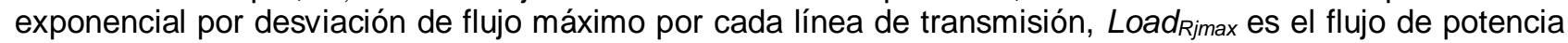
máximo permitido por una línea de transmisión $j$ en p.u, $\operatorname{Load}_{R j, t}$ es el flujo de potencia por una línea de transmisión $j$ en p.u en el periodo $t ; \lambda_{\text {l }}$ es una constante de penalización exponencial por desviación de pérdidas de potencia activa máxima, $\operatorname{Loss}_{\text {Ref }}$ es la referencia de pérdidas de potencia activa máxima diaria y $P_{\text {lossd }}$ son las pérdidas de potencia activa diarias.

En la figura 1 se ilustran las subfunciones empleadas para evaluar tensiones en los nodos, flujos por las líneas y pérdidas de potencia durante las 24 horas del día. Es importante resaltar que las subfunciones que se describen más adelante para restricciones de maniobras de taps de transformadores, como también para los límites de maniobras diarias en capacitores tienen una estructura similar a las ilustradas en la figura 1. Esto garantiza que una solución factible y óptima (desde el punto de vista de la meta en pérdidas) tiene un valor igual a uno.

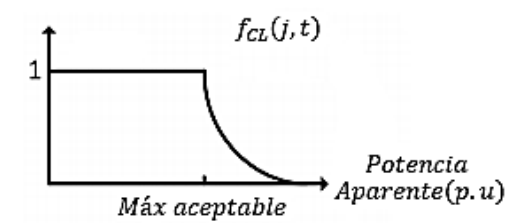

a)

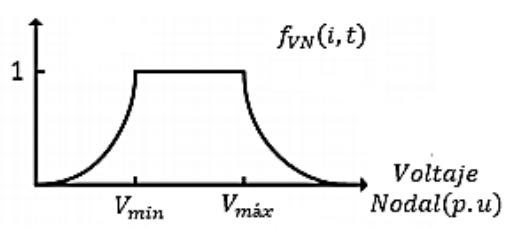

b)

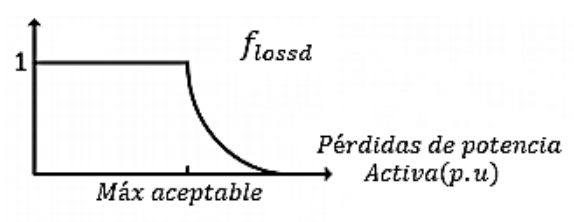

c)

Fig. 1: a) Límites de flujo de potencia en el tiempo $t$; b) Límites de voltaje en las barras de carga el tiempo $t$; y c) Límite de las pérdidas de potencia activa diarias. 


\section{Restricciones interhorarias para maniobras de taps de transformadores}

Para los transformadores con cambio de tap se deben tener en cuenta las restricciones (5) a (9). En La restricción $(5), f_{C T}(k, t)$ indica una subfunción de costos de compensación por maniobras realizadas en el transformador késimo, en el periodo $t$, donde $\lambda_{C T}, C_{M a x h, T, k}$ y $C_{O T}(k, t)$ son la constante de penalización exponencial por desviación de maniobras máximas permitidas en los transformadores, costo máximo de maniobras horarias permitidas en el transformador k-ésimo y el costo de operación horaria por maniobras realizadas en el transformador k-ésimo en el periodo t, respectivamente. Las expresiones (6) y (7) indican el costo máximo de maniobras horarias permitidas en el transformador k-ésimo y el costo de operación horaria por maniobras realizadas en el transformador k-ésimo en el periodo $\mathrm{t}$, donde $C T_{k}$ es el costo de operar el transformador k-ésimo, $T_{\operatorname{maxh}, k}$, es el número máximo de maniobras permitidas para el transformador k-ésimo, $N T_{k, t}$ es el número de maniobras realizadas por el transformador k-ésimo en el periodo t, $S_{\text {base }}$ y $P_{\text {base }}$ son la potencia activa y aparente base, respectivamente. En las expresiones (8) y (9) Tapk,t y Tapk,t-1 corresponden al tap del transformador k-ésimo en el periodo $t$ y en el periodo t-1, $\alpha_{k, t}$ es el valor en p.u de la ubicación del tap del transformador k-ésimo en el periodo t, y $P_{k}$ es el paso del cambio de tap del transformador k-ésimo.

$$
\begin{aligned}
& f_{C T}(k, t)=\left\{\begin{array}{c}
e^{\left\{\lambda_{C T}\left[C_{\text {Maxh },, k}-C_{O T}(k, t)\right]\right\},} C_{O T}(k, t)>C_{\text {Maxh }, T, k} \\
1, C_{O T}(k, t) \leq C_{M a x h, T, k}
\end{array}\right\} \\
& C_{\text {Maxh }, T, k}=\frac{C T_{k} * \operatorname{Tmax}_{h, k}}{P_{\text {base }}} \\
& C_{O T}(k, t)=\frac{C T_{k} * N T_{k, t}}{P_{\text {base }}} \\
& P_{\text {base }}=0.9 * S_{\text {base }}[M W] \\
& N T_{k, t}=\left|\operatorname{Tap}_{k, t}-\operatorname{Tap}_{k, t-1}\right|, k=1, \ldots, T, t=1, \ldots, 24 h . \\
& \alpha_{k, t}=1+P_{k} * \operatorname{Tap}_{k, t}, \quad k=1, \ldots, T, t=1, \ldots, 24 h .
\end{aligned}
$$

\section{Límite diario de maniobras de taps en transformadores}

Para mantener las condiciones del número de operaciones de los taps del transformador durante su vida útil se define un número máximo de maniobras diarias para cada transformador. Esto para que la optimización de la potencia reactiva no afecten la vida útil de los transformadores. Para esto se emplean las ecuaciones dadas por (10) y (11).

$$
\begin{aligned}
& f_{C T d}(k)=\left\{\begin{array}{c}
e^{\left\{\lambda_{C T}\left[C_{\text {Maxd }, T, k}-\sum_{t=1}^{24} C_{O T}(k, t)\right]\right\}}, \sum_{t=1}^{24} C_{O T}(k, t)>C_{\text {Maxd }, T, k} \\
1, \quad \sum_{t=1}^{24} C_{O T}(k, t) \leq C_{\text {Maxd }, T, k}
\end{array}\right\} \\
& C_{\text {Maxd }, T, k}=\frac{C T_{k} * \text { Tmax }_{d, k}}{P_{\text {base }}}
\end{aligned}
$$

Donde $f_{C T d}(k)$ corresponde a la subfunción de costos diario debido a la operación del transformador k-ésimo, $C_{\text {maxd,T,k }}$ es el costo máximo de maniobras diarias permitidas para el transformador k-ésimo y $T_{\text {maxd, } k}$ es el número máximo de maniobras diarias permitidas para el transformador k-ésimo.

\section{Límite de maniobras diarias en bancos de capacitores}

En los elementos de compensación reactiva en derivación también se debe establecer un número máximo de maniobras de conexión diarias. Para ello se emplean las ecuaciones (12), (13), (14) y (15). En (12) $f_{C C d}(l)$ es la subfunción de costos diarios debido a la operación del banco de capacitores l-ésimo, $\lambda_{c c}$ es una constante de penalización exponencial por desviación de maniobras máximas permitidas en los bancos de capacitores, $C_{\text {maxd, } C, l}$ es el costo máximo de maniobras diarias permitidas para el banco de capacitores l-ésimo. En la expresión (13), $C C_{\text {l, }}$ es el costo de operar el banco de capacitores I-esimo, $C_{\text {maxd, }}$ es el número máximo diario de maniobras permitidas para el banco de capacitores I-ésimo. En (14) $C_{o c}(l, t)$, es el costo de maniobras horarias realizadas por el banco de capacitores l-ésimo en el periodo t, $N C_{l, t}$ es el número de maniobras horarias realizadas por el banco de capacitores l-ésimo en el tiempo t. En (15) $C_{l, t}, C_{l, t-1}$ y $\Delta C_{-}$corresponden a las capacidades de potencia reactiva inyectadas por el banco de capacitores l-ésimo, en el tiempo t y en el tiempo t-1, y el paso de inyección de potencia del banco de capacitores l-ésimo, respectivamente. 


$$
\begin{aligned}
& f_{C C d}(l)=\left\{\begin{array}{c}
e^{\left\{\lambda_{C C}\left[C_{\text {Maxd.C, }, l}-\sum_{t=1}^{24} C_{O C}(l, t)\right]\right\}}, \sum_{t=1}^{24} C_{O C}(l, t)>C_{\text {Maxd. }, l} \\
1, \quad \sum_{t=1}^{24} C_{O C}(l, t) \leq C_{\text {Maxd.C, },}
\end{array}\right\} \\
& C_{M a x d, C, l}=\frac{C C_{l} * \operatorname{Cmax}_{d, l}}{P_{\text {base }}} \\
& C_{O C}(l, t)=\frac{C C_{l} * N C_{l, t}}{P_{\text {base }}} \\
& N C_{l, t}=\frac{\left|C_{l, t}-C_{l, t-1}\right|}{\Delta C}, \quad l=1, \ldots, C, \quad t=1, \ldots, 24 h .
\end{aligned}
$$

\section{MÉTODO DE SOLUCIÓN}

El modelo matemático descrito en (1)-(15) es no lineal y no convexo. Este tipo de problemas se deben abordar con técnicas de optimización metaheurísticas (Villa-Acevedo et al., 2019). En este caso, se seleccionó el MVMO como método de solución. La característica novedosa del MVMO es el uso de una función de asignación o mapeo especial aplicada para la mutación de las nuevas generaciones, basado en la media y la varianza de la mejor población encontrada hasta el momento. La forma y localización de la curva de mapeo se ajustan en función del proceso de búsqueda. EI MVMO actualiza la solución candidata con la mejor solución encontrada en cada iteración. La versión de enjambre de este algoritmo, combina las bondades de la función de mapeo con la inteligencia de enjambre, lo cual vuelve el algoritmo más robusto a problemas de estancamiento en mínimos locales (Erlich, 2010). En la figura 2 se ilustra el diagrama de flujo del algoritmo propuesto.

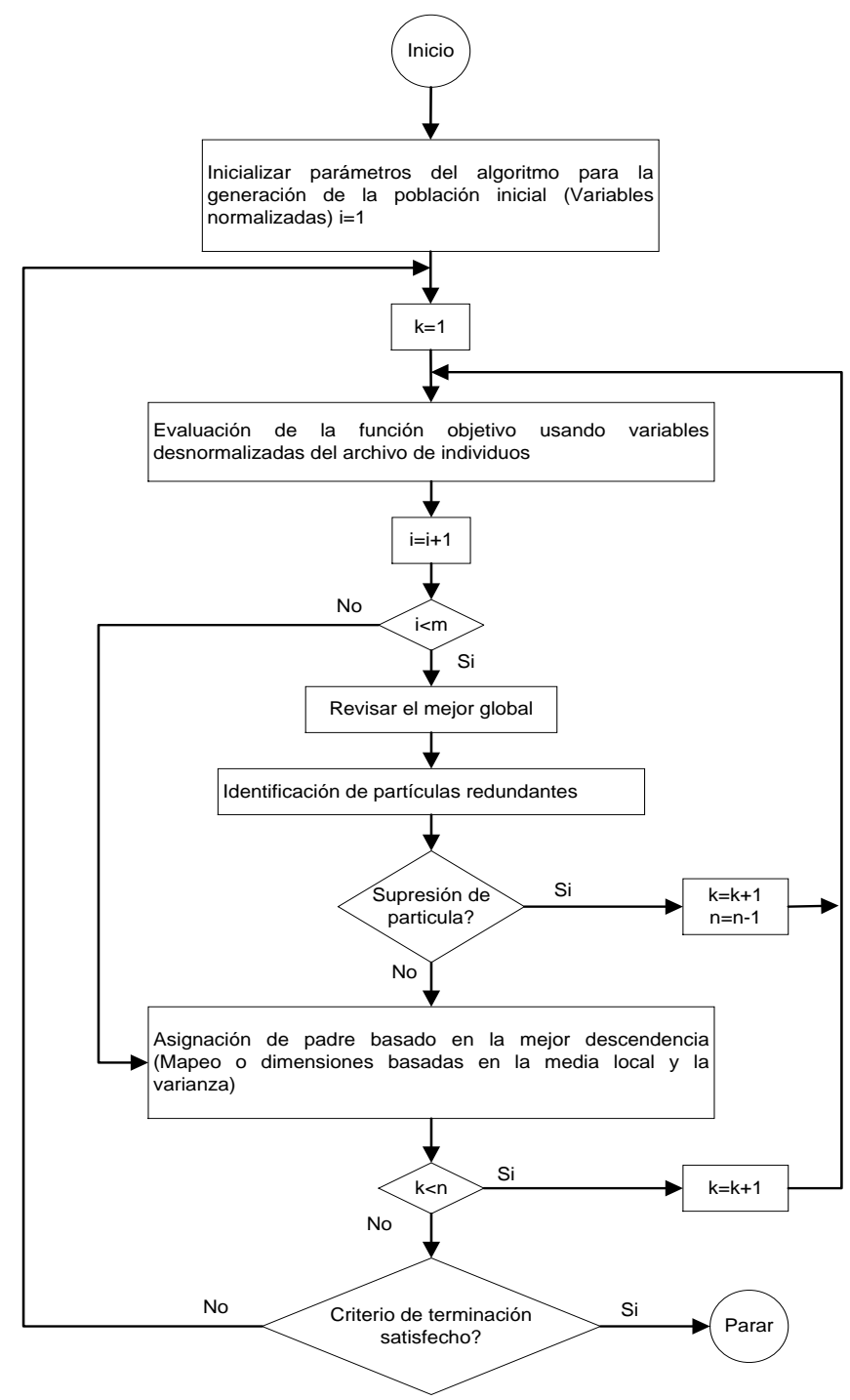

Fig. 2: Diagrama de flujo del algoritmo de solución propuesto. 
Como en muchos algoritmos evolutivos, el MVMO opera sobre un conjunto de soluciones. El espacio de búsqueda interno de todas las variables en el MVMO está restringido al intervalo [0,1]. Por lo tanto, los límites mínimos y máximos de las variables deben ser normalizados en este rango. Durante cada iteración no es posible que ningún componente del vector de solución viole los límites correspondientes. Para lograr este objetivo se desarrolló una función de mapeo especial. Las entradas de esta función son la media y varianza de las mejores soluciones que el MVMO ha descubierto hasta el momento. La salida de esta función de mapeo siempre está en el rango [0,1], evitando que ocurra la violación de los límites de variables durante el proceso de búsqueda. La forma y ubicación de la curva de mapeo se ajustan de acuerdo con el progreso de la búsqueda y el MVMO actualiza la solución candidata alrededor de la mejor solución en cada paso de la iteración. Así mismo, el MVMO es capaz de buscar alrededor de la mejor solución local con una posibilidad pequeña de quedar atrapado en los óptimos locales. Esta característica fue mejorada con la estrategia para el manejo de cero varianza (Cepeda et al., 2012).

\section{Evaluación de la aptitud y manejo de restricciones}

En este caso se aplica la prueba chi-cuadrado para cada solución candidata, la factibilidad de la solución es revisada y se asigna un valor a la función de aptitud. Se considera que un individuo es mejor si su aptitud es menor. El enfoque de penalización estática es utilizado para el manejo de las restricciones. Dado que las variables de control son auto limitadas, todas las variables dependientes son restringidas aplicando la función integrada de aptitud como sigue:

$$
\min f^{\prime}=f+\sum_{i=1}^{n} v_{i} \max \left[0, g_{i}\right]^{\beta}
$$

Donde $f$ es la función objetivo original, $n$ es el número de restricciones, $\beta$ es el orden del término de penalización, vi es el coeficiente de penalización de la $i$-ésima restricción y $g$ representa la restricción de desigualdad.

\section{Función de mapeo mejorada}

Esta función transforma una variable $x_{i}^{*}$ modificada aleatoriamente con distribución unitaria a otra variable $x_{i}$, que se concentra alrededor del valor medio. El nuevo valor de la i-ésima variable está dada por (17).

$$
x_{i}=h_{x}+\left(1-h_{1}+h_{0}\right) x_{i}^{*}-h_{0}
$$

Donde $h_{x}, h_{1}$ and $h_{0}$ son las salidas de la función de mapeo basada dadas en (18)

$$
h_{x}=h\left(x=x_{i}^{*}\right), h_{0}=h(x=0), h_{1}=h(x=1)
$$

La función de mapeo está parametrizada como se indica en (19)

$$
h\left(\bar{x}_{i}, s_{1}, s_{2}, x\right)=\bar{x}_{i}\left(1-e^{-x s_{1}}\right)+\left(1-\overline{x_{i}}\right) e^{-(1-x) s_{2}}
$$

Donde $s_{1}$ y $s_{2}$ son factores de forma que permiten variaciones asimétricas de la función de mapeo. El factor de forma está calculado por (20)

$$
s_{i}=-\ln \left(v_{i}\right) f_{s}
$$

En este caso, $f_{s}$ es un factor de escala que permite el control del proceso de búsqueda durante la iteración, mientras $\overline{x_{i}}$ y $v_{i}$ son la media y la varianza del archivo de la solución, respectivamente. En (Cepeda et al., 2012) se pueden consultar los detalles de ajuste de este método.

\section{Archivo de solución}

El archivo de solución constituye el conocimiento básico del algoritmo para guiar la dirección de la búsqueda. Por lo tanto, los $n$ mejores individuos que el MVMO ha encontrado hasta el momento son guardados en el archivo. El valor de aptitud para cada individuo también se guarda. Las siguientes reglas se ajustan para comparar los individuos generados en cada iteración y las soluciones existentes archivadas con el fin de evitar la pérdida de buenas soluciones: (i) cualquier solución factible se prefiere a cualquier solución inviable, (ii) entre dos soluciones factibles, se prefiere la que tenga mejor valor de aptitud, (iii) entre dos soluciones 
inviables, se prefiere la que tenga menor valor de aptitud. Se realiza la actualización solo si el nuevo individuo es mejor que aquellos que están en el archivo. El tamaño del archivo es fijo para todo el proceso. Los individuos archivados son organizados dinámicamente de modo que el primero siempre es el mejor. Las soluciones factibles son ubicadas en la parte alta del archivo. Estas soluciones son organizadas de acuerdo a sus valores de aptitud. Las soluciones inviables son organizadas de acuerdo a su aptitud y ubicadas en la parte más baja del archivo. Una vez el archivo esté completo con $n$ soluciones factibles, cualquier solución de candidato inviable no tiene oportunidad de ser guardada en el archivo.

\section{Nueva generación y criterio de parada}

La primera solución posicionada en el archivo (la mejor hasta el momento), se denota como xbest y es asignada como el padre. Para generar la descendencia el MVMO busca el valor medio guardado en el archivo de las soluciones para encontrar la mejor solución sólo en $m$ direcciones seleccionadas. Esto significa que sólo estas dimensiones de la descendencia serán actualizadas mientras que las $D-m$ dimensiones restantes toman los valores correspondientes del xbest. El proceso de búsqueda del MVMO termina con base en un número predeterminado de evaluaciones de aptitud.

\section{Versión de enjambre del MVMO}

La versión de enjambre del MVMO inicia con $n$ partículas o soluciones candidatas, cada una con su propio archivo de soluciones y su función de mapeo. En el proceso, cada solución candidata ejecuta $m$ pasos para identificar un conjunto óptimo de soluciones independientes. Posteriormente, las partículas intercambian información. En algunos casos, algunas partículas están muy cerca unas de otras, lo que significa que hay información redundante; esto se soluciona descartando las partículas redundantes. Se define una mejor solución local y global. También se calcula la distancia normalizada entre cada partícula a las mejores soluciones locales y globales.

Para una partícula descartada del proceso se usa su distancia normalizada, y si es menor que un cierto umbral predefinido, se tiene lo siguiente: si ese umbral es cero, se tienen en cuenta todas las partículas en todo el proceso; de lo contrario, si el umbral es uno, se descartan todas las partículas excepto las de la mejor solución global. Tras una evaluación independiente, y si la partícula se sigue considerando, su búsqueda se dirigirá hacia la solución global asignando la mejor global en lugar de la mejor solución local, como padre. Los pasos restantes son idénticos a los del MVMO clásico. Una descripción detallada de la variante de enjambre del MVMO puede ser consultada en (Rueda et al., 2012).

\section{RESULTADO Y DISCUSIÓN}

Para validar el desempeño del modelo propuesto se utilizó el sistema de prueba IEEE de 30 barras, al cual se incorporaron 9 bancos de capacitores como se indica en (Radosavljevic, 2016). Inicialmente se define un horizonte temporal de $24 \mathrm{~h}$, para las cuales se establecen demandas de potencia activa y reactiva. En la Tabla 1 se indican las demandas máximas en cada nodo donde Pd y Qd indican las demandas de potencia activa y reactiva en MW y MVAr, respectivamente. Con base en estos datos se consideran diferentes curvas de demanda para cada tipo de usuario como se indica en (Londoño, 2020). El modelo propuesto se implementó en Matlab usando como base el software Matpower (Zimmerman et al., 2011). Para el MVMO consideraron 40 partículas y un máximo de 40000 evaluaciones. Los detalles de su implementación se encuentran disponibles en (Londoño, 2020).

Tabla 1: Caracterización de nodos de carga para el sistema IEEE de 30 barras (demandas máximas).

\begin{tabular}{|c|c|c|l|c|c|c|c|}
\hline Barra & Pd & Qd & Tipo & Barra & Pd & Qd & Tipo \\
\hline 2 & 21.7 & 12.7 & Industrial & 17 & 9 & 5.8 & Residencial \\
\hline 3 & 2.4 & 1.2 & Industrial & 18 & 3.2 & 0.9 & Residencial \\
\hline 4 & 7.6 & 1.6 & Industrial & 19 & 9.5 & 3.4 & Residencial \\
\hline 7 & 22.8 & 10.9 & Industrial & 20 & 2.2 & 0.7 & Residencial \\
\hline 8 & 30 & 30 & Comercial & 21 & 17.5 & 11.2 & Residencial \\
\hline 10 & 5.8 & 2 & Comercial & 23 & 3.2 & 1.6 & Residencial \\
\hline 12 & 11.2 & 7.5 & Comercial & 24 & 8.7 & 6.7 & Residencial \\
\hline 14 & 6.2 & 1.6 & Comercial & 26 & 3.5 & 2.3 & Residencial \\
\hline 15 & 8.2 & 2.5 & Comercial & 29 & 2.4 & 0.9 & Residencial \\
\hline 16 & 3.5 & 1.8 & Residencial & 30 & 10.6 & 1.9 & Residencial \\
\hline
\end{tabular}


Una de las características que distinguen el abordaje propuesto de otros métodos es el manejo de restricciones. Como se indicó en la figura 1, las subfunciones que se utilizan para hacer que se cumplan las restricciones deben parametrizarse con los lambas. En este caso, los factores de penalización de cada una de las subfunciones que componen la función aptitud total son iguales a 0.05; exceptuando la subfunción de flujos máximos por líneas de transmisión que es igual a 0.005 haciendo esta consideración menos restrictiva en el espacio de búsqueda. Los valores de lambdas para las subfunciones planteadas fueron establecidos mediante pruebas de sensibilidad realizadas al modelo. Igualmente, se definen el costo por maniobra de los cambiadores de taps de los transformadores $\left(C T_{k}\right)$ igual a $6 \$$ /maniobra, y el costo por maniobra para bancos de capacitores $\left(C C_{l}\right)$ igual a $4 \$$ /maniobra. En este artículo una maniobra corresponde al movimiento realizado al pasar de un tap a otro en los transformadores, o el movimiento realizado por el interruptor de un banco de capacitores para pasar de una inyección de potencia reactiva de una capacidad, a otra inyección de mayor o menor capacidad. Los valores de $C T_{k}$ y $C C_{l}$ son factores de peso asociados al costo por maniobra para transformadores y capacitores, el cual es mayor para los transformadores, debido a que los transformadores tienen un rol más activo dentro de la operación de los sistemas de potencia.

Para el método de manejo de las restricciones que se propone en este artículo se debe definir una meta de pérdidas. Está meta se establece como un porcentaje de la potencia generada en el sistema. Para este caso se toma el $1.77 \%$ de la generación del sistema. Para el caso base del sistema IEEE de 30 barras se tiene un valor de pérdidas antes de optimizar de $65.36 \mathrm{MW}$ en demanda máxima y la meta de pérdidas para el proceso de optimización se define en $59.5 \mathrm{MW}$. Este valor se obtuvo mediante un análisis de sensibilidad, que estimó el valor a partir del cual no se presenta una reducción de pérdidas notoria, en función del cambio en el número de maniobras diarias de transformadores y bancos de capacitores.

El límite de maniobras diarias de los transformadores fue establecido tomando como base un máximo 500000 maniobras cada 12 años como indican los fabricantes (Londoño, 2020). Lo anterior indica un número de operaciones máximo diario de 114. Para las operaciones diarias de los bancos de capacitores, se toma como criterio el número de maniobras permitidas para los interruptores de las unidades de compensación capacitiva. En este caso se tiene un valor de 20000 maniobras por cada unidad, para un periodo de mantenimiento preventivo de 20 años. El número máximo de maniobras diarias para los interruptores de los capacitores es igual a 2.74. Los límites de tensión mínimos y máximos en las barras de carga son 1.1 y 0.9 en por unidad respectivamente. En la Tabla 2 se ilustran los demás límites para las variables de control considerando una potencia base de 100 MVA. Se realizaron varias corridas con el algoritmo propuesto en un equipo con un procesador AMD Ryzen de 2.3GHz y 20 GB de memoria RAM. El tiempo de cómputo promedio fue de 7000.53 segundos.

Tabla 2: Límites de las variables de control para sistemas IEEE de 30 barras.

\begin{tabular}{|c|c|c|c|c|c|c|c|}
\hline $\begin{array}{c}\text { Variables de } \\
\text { control }\end{array}$ & $V_{G}{ }^{\max }$ & $V_{G}{ }^{\min }$ & $T_{i}{ }^{\min }$ & $T_{i}{ }^{\max }$ & $Q_{C}{ }^{\max }$ & $Q_{C}{ }^{\min }$ & $Q_{C}{ }^{\text {Paso }}(\Delta C)$ \\
\hline Límites & 1.1 & 0.95 & 0.9 & 1.05 & 0.05 & 0 & 0.01 \\
\hline
\end{tabular}

\section{Tensiones en nodos de generación}

En la figura 3 se ilustran las tensiones en el nodo 1 para tres corridas del algoritmo seleccionadas al azar. Se puede evidenciar una mejoría en los valores de las tensiones en el periodo bajo estudio. Se pudo comprobar que en este caso el $93 \%$ de los valores de tensión se encuentran por encima de los valores iniciales del caso base. Un comportamiento similar se pudo verificar en los otros nodos de generación. Es importante resaltar también que se presentan corridas para las cuales se encuentran valores de tensión en algunas barras por debajo del caso base, pero siempre en el rango operativo permisible.

\section{Tensiones en nodos de carga}

Al revisar las variaciones de tensión horarias se encuentra que en el 100\% de los casos, las tensiones en los nodos comercial y residencial aumentan después del despacho óptimo de potencia reactiva. Para el caso de los nodos con carga industrial, esto ocurre el $89.17 \%$ de las veces. A manera ilustrativa la figura 4 muestra el comportamiento de las tensiones en el nodo 7 (carga industrial). Por otro lado, se presentan corridas para las cuales se encuentran valores de tensión por debajo del caso base; sin embargo, las mismas se encuentran en el rango permisible.

\section{Variación en taps de transformadores}

En los resultados se observó un comportamiento similar en la variación de los taps de transformadores. En la figura 5 se presenta de forma ilustrativa la variación de taps del transformador 1, ubicado entre los nodos 69 . También se tiene que la restricción de número de operaciones horarias y diarias se cumple como se indica en la figura 6, las cuales fueron estimadas en un límite máximo de 5 operaciones horarias y 30 diarias. 


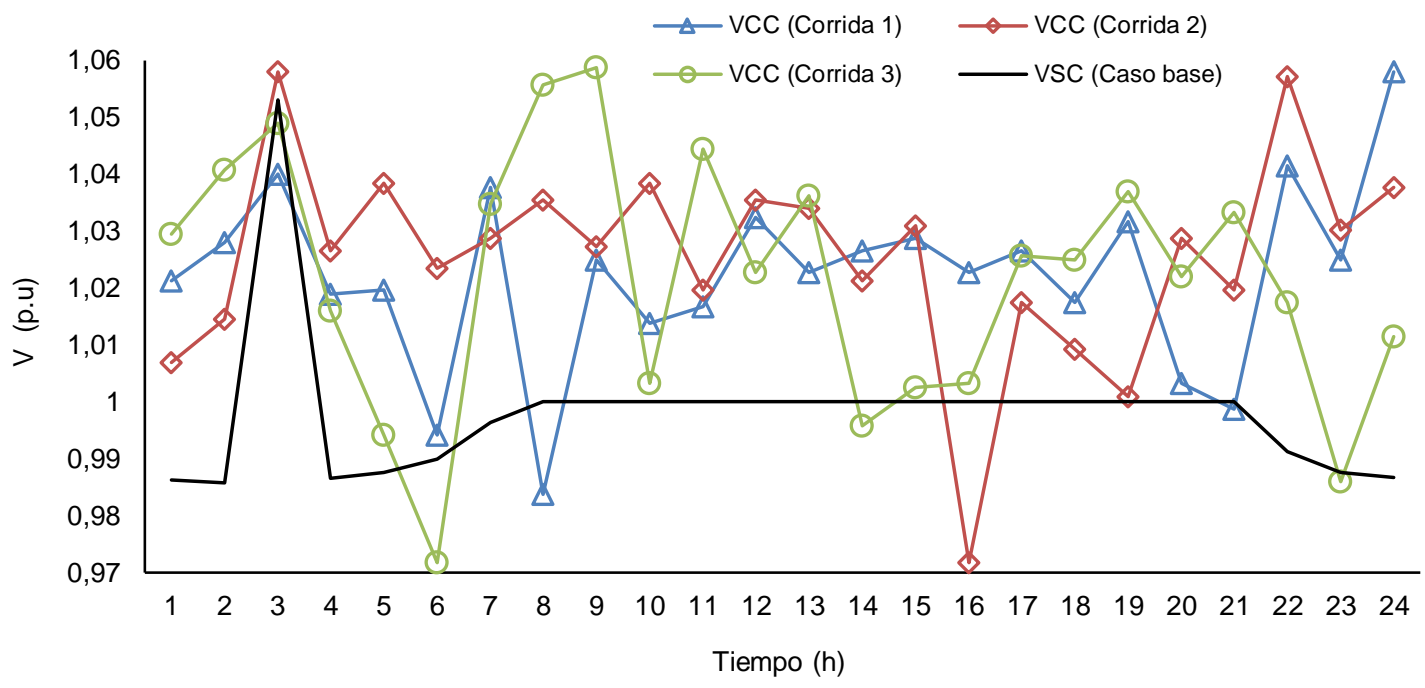

Fig. 3: Tensiones del generador ubicado en la barra 1 para diferentes corridas del algoritmo.

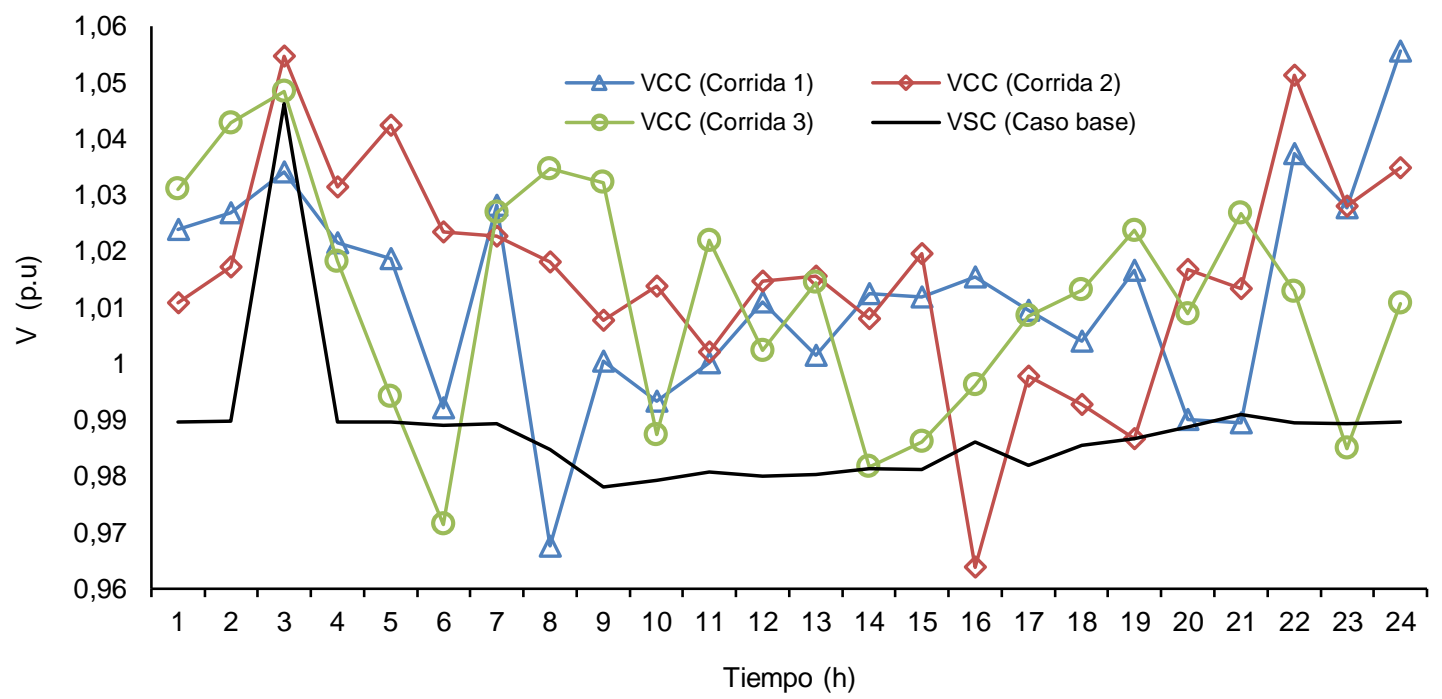

Fig.4: Voltaje en el nodo 7 (carga industrial) del sistema IEEE de 30 barras.

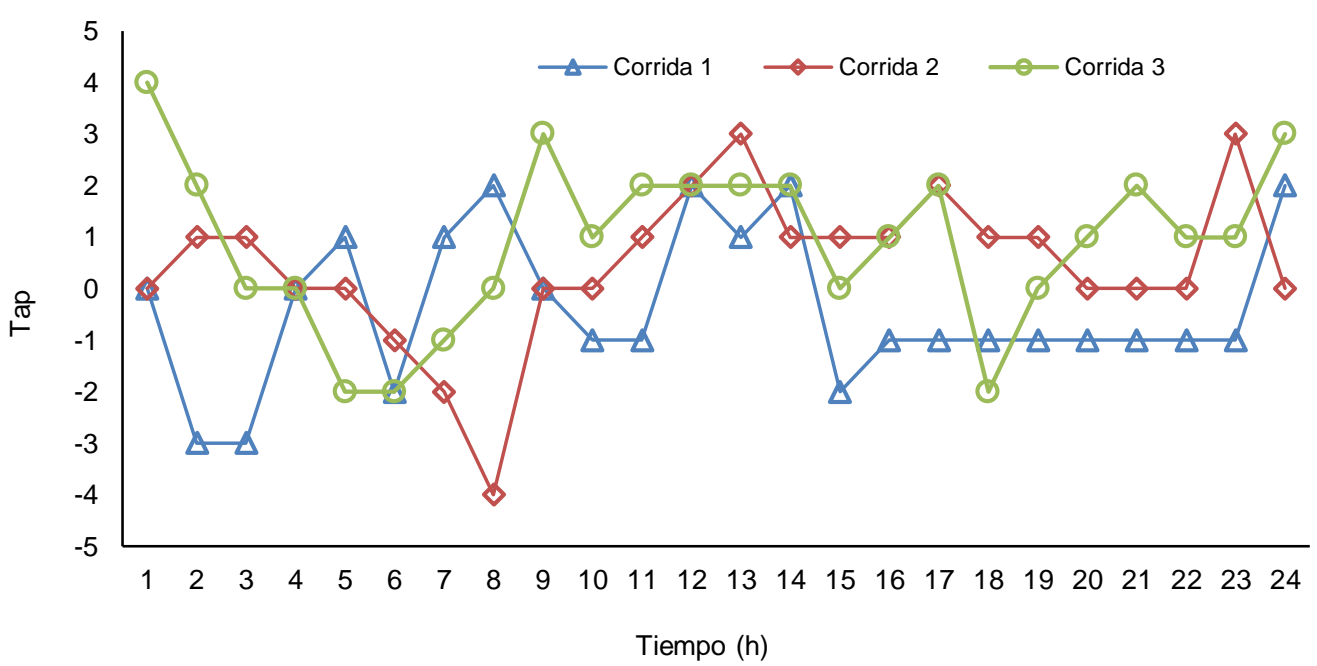

Fig. 5: Variación horaria de taps del transformador 1 (nodos 6-9) para el sistema IEEE de 30 barras. 


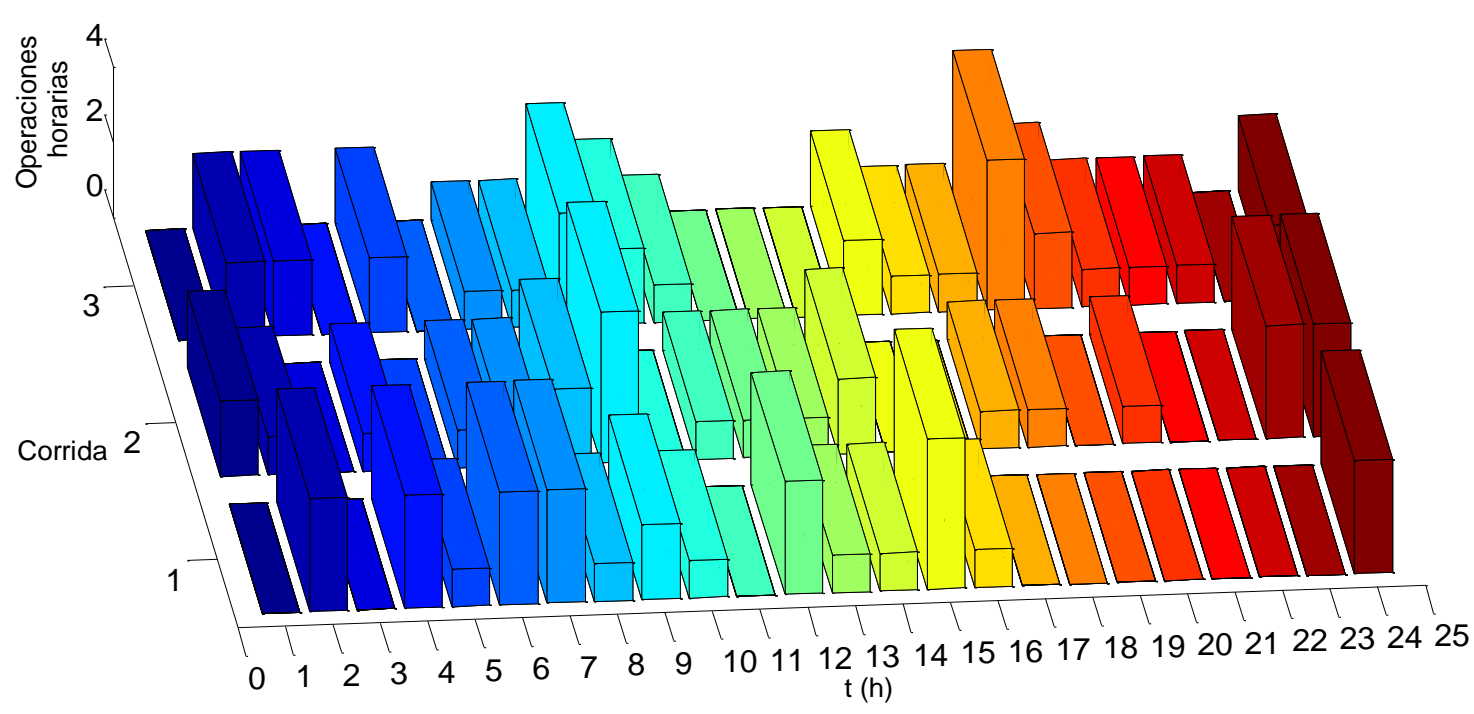

Fig. 6: Operaciones horarias del transformador 1 (nodos 6-9) para el sistema IEEE de 30 barras.

\section{Operaciones horarias y diarias de los bancos de capacitores}

En la figura 7 se presenta la variación de la potencia reactiva inyectada por el banco de capacitores ubicado en la barra 20. Se puede observar que la inyección de potencia reactiva se encuentra en el rango de 0 a 4 MVAr. En el caso de los bancos de capacitores no se estableció un límite horario al número de operaciones, dado que estos operan pocas veces en el día. Sin embargo, los resultados de las simulaciones presentan un rango de operación horario para cada banco de capacitores de 0 a 3 operaciones entre periodos consecutivos.

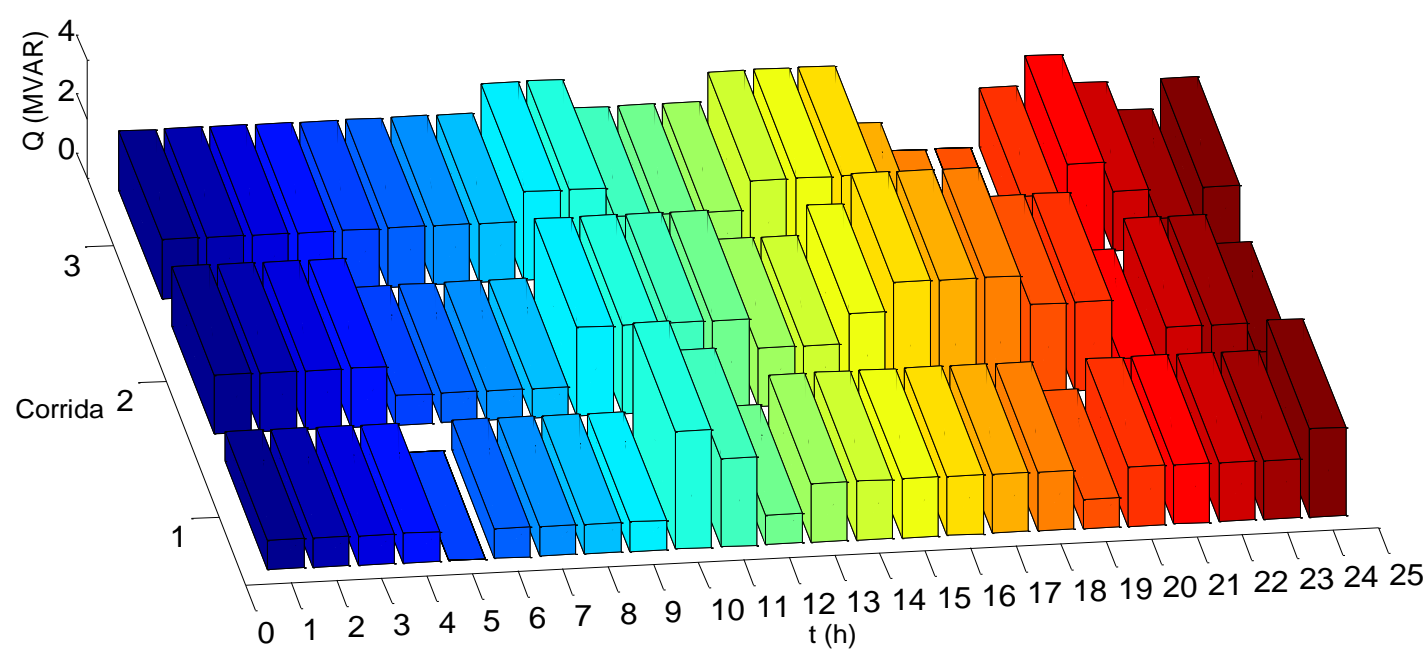

Fig. 7: Variación de potencia reactiva del banco de capacitores ubicado en el nodo 20.

\section{Convergencia del algoritmo}

En la figura 8 se ilustra la convergencia del algoritmo para tres corridas. Se puede observar que en todos los casos la solución encontrada por el algoritmo presenta la misma función de aptitud, lo cual evidencia la reproducibilidad de las soluciones obtenidas. Por otro lado, la figura 9 ilustra el porcentaje de cargabilidad de las líneas con mayor flujo de potencia del sistema. Se puede observar que el algoritmo garantiza que no se violen los límites de cargabilidad de las líneas. Los resultados obtenidos anteriormente evidencian la aplicabilidad del modelo y método de solución propuesto. Uno de los factores a tener en cuenta para la aplicación a sistemas de gran tamaño es el tiempo computacional; en este caso fue de menos de dos horas, pero puede ampliarse a varias horas para otro tipo de sistemas. El tiempo computacional se puede reducir de forma significativa usando paralelización o incluso mediante la implementación de la metodología en otro software de optimización. Si se cuenta con un pronóstico confiable, las fuentes de generación renovables se pueden considerar de forma directa en el modelo en los nodos de generación. Sin embargo, se puede llevar a cabo así mismo un modelamiento estocástico. Esta última opción está por fuera del alcance del presente artículo. 


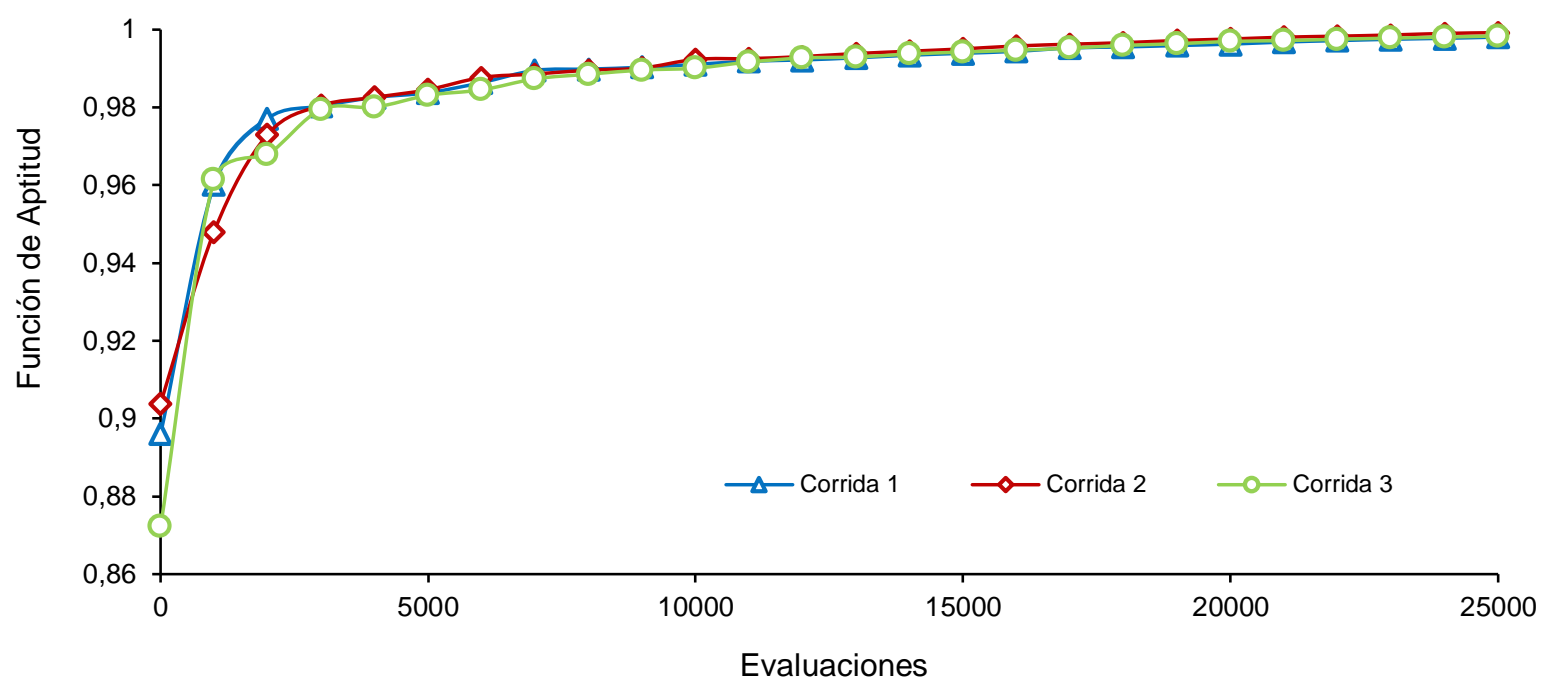

Fig. 8: Convergencia del algoritmo para tres corridas

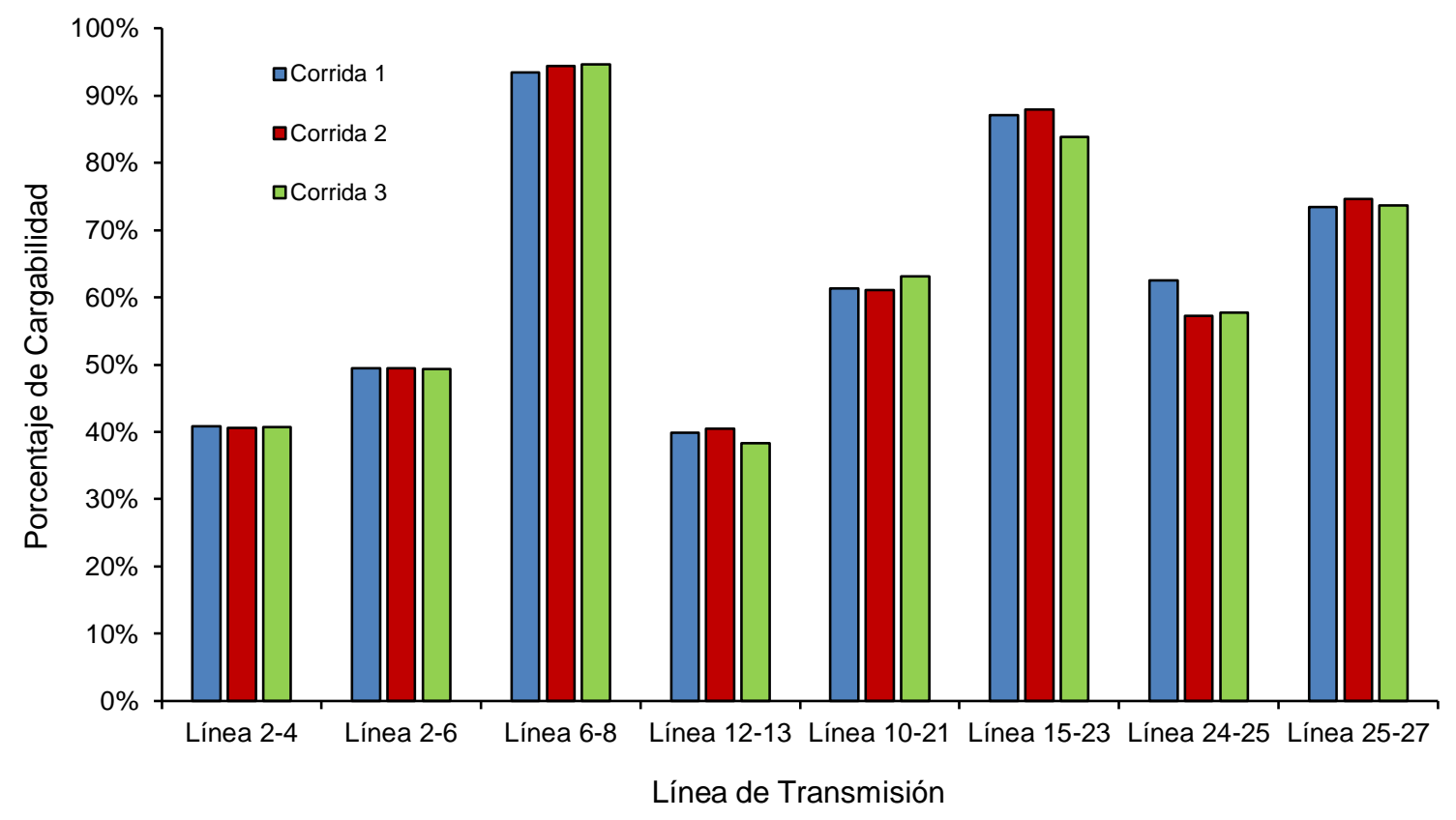

Fig. 9: Porcentaje de cargabilidad de las líneas con mayor flujo de potencia

\section{CONCLUSIONES}

En este artículo se propone un nuevo modelo matemático para solucionar el DOPRM, en el cual se tienen en cuenta las restricciones operativas del sistema de potencia y de los elementos de maniobra que realizan el control de potencia reactiva. La formulación matemática propuesta para el manejo de las restricciones tanto operativas como de maniobra, permiten garantizar la convergencia a una solución del problema de optimización, esto sucede cuando se llega a un valor prefijado de la función de aptitud, al llegar al valor de la aptitud deseado se garantiza el cumplimiento de las metas propuestas y restricciones operativas establecidas para el sistema. El modelo matemático propuesto para resolver el problema del DOPRM encuentra de forma rápida soluciones viables evitando realizar evaluaciones adicionales, lo que se significa un ahorro en tiempo y recursos de simulación. En el modelo propuesto se busca no afectar la vida útil de los elementos de maniobras de los transformadores e interruptores de elementos de compensación, para lo cual se incluyen restricciones de maniobras interhorarias y diarias para los transformadores y elementos de compensación reactiva. La aplicación del algoritmo MVMO para la solución del DOPRM presenta una convergencia temprana cuando se realizan varias ejecuciones del MVMO. Para todas las soluciones encontradas por el MVMO, esto es, cuando alcanzan la meta propuesta para la función objetivo cumpliendo con las restricciones, se presentan mejoras notables en los perfiles de tensión de los nodos y en la reducción de maniobras en taps de los transformadores y conexión de elementos de compensación. 


\section{REFERENCIAS}

Cepeda, J.C., Rueda, J.L., y Erlich , I., Identification of dynamic equivalents based on heuristic optimization for smart grid applications, http://doi.org/10.1109/CEC.2012.6256493, IEEE World Congress on Computational Intelligence, 10-15, (2012).

Davoodi, E., Babaei, E., Mohammadi-Ivatloo, B., y Rasouli, M., A novel fast semidefinite programming-based approach for optimal reactive power dispatch, http://dx.doi.org/10.1109/TII.2019.2918143, IEEE Transactions on Industrial Informatics, 16(1), 288-298 (2020).

Erlich, I., Venayagamoorthy, G.K., y Worawat, N., A mean variance optimization algorithm, http://doi.org/ 10.1109/CEC.2010.5586027, IEEE Congress on Evolutionary Computation, (2010).

Gutiérrez, D., López, J.M., y Villa W.M., Metaheuristic techniques applied to the optimal reactive power dispatch: a review, http://doi.org/10.1109/TLA.2016.7530421, IEEE Latin America Transactions, 14(5), 2253-2263, (2016).

Gutiérrez, D., Villa, W.M., y López, J.M., Flujo óptimo reactivo mediante optimización por enjambre de partículas, http://dx.doi.org/10.4067/S0718-07642017000500020, Información Tecnológica, 28(5), 215-224 (2017).

Huang, J., Li, Z., y Wu, Q.H., Fully decentralized multiarea reactive power optimization considering practical regulation constraints of devices, http://doi.org/10.1016/j.ijepes.2018.08.045, Int. J. Electr. Power Energy Syst., 105, 351-364, (2019).

Jamal R., Men, B., y otros cuatro autores, Application of Shannon entropy implementation into a novel fractional particle swarm optimization gravitational search algorithm (FPSOGSA) for optimal reactive power dispatch problem, http://doi.org/10.1109/ACCESS.2020.3046317, IEEE Access, 9(1) 2715-2733 (2020).

Londoño, D.C., Modelado y solución del despacho óptimo reactivo multiperiodo mediante una técnica de optimización metaheurística, Tesis de Maestría, Universidad de Antioquia (2020).

López, J., Contreras, J., y Mantovani, J.R.S., Reactive power planning under conditional-value-at risk assessment using chance-constrained optimization, http://doi.org/10.1049/iet-gtd.2014.0224, IET Gener.Transm. Distrib., 9(3), 231-240, (2015).

Mohseni-Bonab, S. M., y Rabiee, A., Optimal reactive power dispatch: a review, and a new stochastic voltage stability constrained multi-objective model at the presence of uncertain wind power generation, http://doi.org/10.1049/ietgtd.2016.1545, IET Gener. Transm. Distrib., 11(4), 815-829, (2017).

Mugemanyi, S., Qu., Z., y otros cuatro autores., Optimal reactive power dispatch using chaotic bat algorithm, http://doi.org/10.1109/ACCESS.2020.2982988, IEEE Access, 8(1), 65830-65867 (2020).

Radosavljevic, J., y Jevtic, M., Solution of optimal reactive power dispatch by a hybrid GSA-SQP algorithm, https://doi.org/10.5755/j01.eie.22.3.15306, Elektronika ir elektrotechnika, 22(3), 3-6, (2016).

Rueda, J.L., Cepeda, J.C., y Erlich, I., Estimation of location and coordinated tuning of PSS based on mean-variance mapping optimization, http://doi.org/10.1109/PESGM.2012.6345025, IEEE Power and Energy Society General Meeting, $1-8,(2012)$.

Singh, R.P., Mukherjee, V., y Ghoshal, S.P., Optimal reactive power dispatch by particle swarm optimization with an aging leader and challenges, http://doi.org/10.1016/j.asoc.2015.01.006, Appl. Soft Comput., 29, 298-309, (2015).

Theologi, A.M., Ndreko, M., y otros tres autores, Optimal management of reactive power sources in far-offshore wind power plants, http://doi.org/10.1109/PTC.2017.7980833, 2017 IEEE Manchester PowerTech., 1-6, (2017).

Villa-Acevedo, W.M., López-Lezama, J.M., y Valencia-Velásquez, J.A., A novel constraint handling approach for the optimal reactive power dispatch problem, http://doi.org/10.3390/en11092352, Energies, 11(9), 1-23, (2018).

Villa-Acevedo, W.M., Rodríguez-Serna, J. M. y Saldarriaga-Loaiza, J. D., Estimación de parámetros del suelo de dos capas usando el algoritmo de optimización de mapeo media-varianza, http://dx.doi.org/10.4067/S071807642019000100299, Información Tecnológica, 30(1), 299-310, (2019).

Yan, W.,Lu, S., y Yu, D.C., A novel optimal reactive power dispatch method based on an improved hybrid evolutionary programming techniques, http://doi.org/10.1109/TPWRS.2004.826716, IEEE Trans. Power Syst., 19(2), 913-918, (2004).

Zhang, L., Tang, W., Liang, J., y Cong, P., Coordinated day-ahead reactive power dispatch in distribution network based on real power forecast errors, http://doi.org/10.1109/TPWRS.2015.2466435, IEEE Trans. Power Syst., 31(3), 2472-2480, (2016).

Zhao, J., Ju, L., Dai, Z., y Chen, G., Voltage stability constrained dynamic optimal reactive power flow based on branchbound and primal-dual interior point method, http://doi.org/10.1016/j.ijepes.2015.05.038, Int. J. Electr. Power Energy Syst., 73, 601-607, (2015).

Zimmerman, R.D., Murillo, C.E., y Thomas, R.J., Matpower: Steady-state operations, planning, and analysis tools for power systems Research and Education, http://doi.org/10.1109/TPWRS.2010.2051168, IEEE Trans. Power Syst., 26(1), 12-19, (2011). 